\title{
Knowledge, Attitude and Practices (KAP) of Sugar Sweetened Beverages (SSBs) Amongst Adolescents in Malaysian Secondary Schools: A cross-Sectional Study
}

NUR ISLAMI MOHD FAHMI TENG ( $\square$ nurislami@uitm.edu.my )

Universiti Teknologi MARA https://orcid.org/0000-0002-6305-8416

Norsham Juliana Nordin

Universiti Sains Islam Malaysia

Nur Liyana Izlin Mohamad Rodi

Universiti Teknologi Mara - Kampus Puncak Alam

Nur Zulaikha Semaon

Universiti Teknologi Mara - Kampus Puncak Alam

\section{Research}

Keywords: sugar-sweetened beverages, body composition, adolescents

Posted Date: July 27th, 2020

DOI: https://doi.org/10.21203/rs.3.rs-46842/v1

License: (c) (i) This work is licensed under a Creative Commons Attribution 4.0 International License.

Read Full License 


\section{Abstract}

Background: This study aims to examine the level of knowledge, attitude and practices (KAP) of adolescents towards sugar-sweetened beverages (SSB) together with the associated factors that determines their KAP.

Methods: This is a cross-sectional study. Data was collected using self-administered questionnaires that consisted of sociodemographic, the KAP for the SSB questionnaire, and the BEVQ questionnaire. The respondents' height, weight, waist circumference and body fat percentage were measured. This study involved 439 adolescents (with a $97.6 \%$ response rate), aged between 13 to 17 years old, in public secondary schools in Selangor, Malaysia.

Results: The results reveal that $35 \%$ of the adolescents were overweight, $26 \%$ had a high waist circumference, and $45 \%$ had a high body fat percentage. Caffeinated drinks and full cream milk were the most frequently consumed SSBs. The KAP score revealed a high attitude (88.4\%), a moderate knowledge $(51.8 \%)$ and a low practice (40.5\%). Those with a higher body fat percentage showed significantly higher attitude scores $(p<0.05)$. Low household income groups (adjusted $\mathrm{OR}=0.51 ; 95 \% \mathrm{Cl}=0.26-0.99$ ), females (adjusted OR=0.41, 95\% $\mathrm{Cl}=0.27-0.62$ ), adolescents aged 16-17 years old (adjusted OR=0.44, 95\% $\mathrm{Cl}=0.22-0.85$ ), and urban areas (adjusted $\mathrm{OR}=1.78,95 \% \mathrm{Cl}=1.16-2.72$ ) demonstrated a significant $(p<0.05)$ positive determinant towards the KAP score.

Conclusion: This study revealed that high awareness of negative health outcomes associated with SSBs among adolescents was not in accord with the level of their lifestyle choices. Future interventions must consider this critical situation so that any knowledge being transferred to the adolescents can be translated into their own practices.

\section{Authorship}

All of the authors participated in the study design. Nur Liyana Izlin \& Nur Zulaikha Semaon collected the data, performed the data analyses, and drafted the manuscript. Nur Islami Mohd Fahmi Teng and Norsham Juliana Nordin were responsible for designed the study, data analyses and critically revising the manuscript. All authors contributed to the writing and approval of the final version of the manuscript.

\section{Background}

Sugar-sweetened beverages (SSBs) are defined as beverages with added sugar, and include sports drinks, sweetened tea and coffee, soft drinks and electrolyte-replacement drinks. The calories contained in SSBs provide little to no nutritional value and less satiety, as compared to solid food. Hence, SSB intake leads to unhealthy weight gain resulting from a high total energy intake, with little nutritional value (1). Globally, SSB intake is the highest amongst adolescents aged between 13 to 20 years (2). The increase of the 
prevalence and degree of obesity amongst adolescents in many populations typically leads to significant public health problems. In Malaysia, the prevalence of obesity has increased from $5.7 \%$ in 2011 , to $11.9 \%$ in 2015 (3). A higher prevalence of obesity in Malaysia was found in urban areas, as compared to rural areas (4). In Metropolitan Kuala Lumpur, the prevalence of overweight and obesity cases was even more worrying, with $34.2 \%$ of obesity cases being amongst young adults(5). The high availability of SSBs, the high density of fast food chain outlets, and poor lifestyle choices, were amongst the factors that contributed to the country's rising problems such as diabetes, obesity and other non-communicable disease (6). In addition, a higher consumption of SSBs was associated with poor oral health, the development of metabolic syndromes, as well as cardio-metabolic risk factors such as type 2 diabetes, later in life (7). The development of type-2 diabetes and excessive weight gain was reported to be strongly related to the consumption of added sugar (8).

Great effort has been made to alarm the population about the hazardous effects of high sugar intake as a major contributing factor to the rise of obesity prevalence and metabolic diseases. However, a report by Amarra et al. (2016) highlighted that the daily intake among the Malaysian population was still above that recommended by the WHO (9). MyHeARTs study in 2016 reported that adolescents in Malaysia were highly susceptible to a high intake of sugar, with the disparity of intakes being based on geographical locations of the adolescents (10). Pengpid \& Peltzer (2020) mentioned that adolescents' intake of SSB, specifically in terms of carbonated soft drinks, is not just associated with metabolic problems, but also with poor mental health (11). The majority of the consumption of daily added sugar by adolescents in Malaysia involved SSBs. They were reported to consume beverages at any time of the day, with the most frequent consumption during breakfast (11).

To date, data in this area is still lacking, and are limited to the trend of intake. It is critical to comprehend in-depth whether the adolescents have sufficient knowledge on SSBs in order to appraise their choice and rate of SSB intake. Our primary objective is to measure the levels and determinants of KAP on SSBs amongst adolescents in urban secondary schools across Malaysia. A predefined self-report questionnaire was adopted to identify the KAPs for SSBs among adolescents. It involves a multistep process that comprises both reporting, as well as the cultural adaptation of these questionnaires. The findings serve as a baseline for developing appropriate intervention measures and raise awareness on the negative impact of SBBs.

\section{Methods}

\section{Sample and study design}

A cross-sectional study was conducted across three secondary schools in Selangor. Selangor is the most developed state in Malaysia, and is divided into nine districts. It is located at the west side of the country. The total population is around 6.53 million, which include three main ethnics, namely, Malay, Chinese and Indian, with Malay being the majority (12). In this study, the participants comprised both male and female students, aged between 13 to 18 years. In the Malaysian education system, adolescents in this age range 
are generally students in secondary school. Participants who had diseases, were on medication, or fall under the 'special needs' category were excluded from the study. A sample size of at least 385 adolescents was determined using Krejcie \& Morgan's equation from a population of 322,164 registered secondary school students in Selangor, with a 95\% confidence interval. Data collection was performed and completed on November 2019.

This study involved a convenience sampling design. A complete list of 261 secondary schools in Selangor was obtained from the Department of Education of Selangor. A total of three schools, each from a different district, were selected. The selection was performed using a multi-staged cluster sampling design. The school with the greatest number of students in each selected district was chosen.

Subsequently, all students who fulfilled the inclusion criteria were invited to participate in the study. The consent form was handed to the participants one day before the data collection was carried out.

Participants consisted of students who attended school on the day of the data collection, and had handed in the signed-off consent form. A total of 450 consented forms were received.

\section{Questionnaire and Anthropometric Measurements}

A set of validated questionnaires was administered to the adolescents. The questionnaire consisted of socio-demographic sections, KAP questionnaires (13), and BEVQ questionnaires, which were adopted from Hedrick et al. (2012) (14).

The KAP questionnaire consists of 20 dichotomous questions ( 10 items for knowledge, 5 items for attitude and 5 items for practice) that require a response of either Yes/No or Agree/Disagree. This questionnaire has been tested for reliability and validity in both the English and Malay languages (15). The overall score was calculated by summing the correct responses by the participants. The maximum score for each domain is equal to the number of items. Furthermore, each domain was categorised into two levels of indicators, 'poor' or 'good', based on the median of results. 'Poor knowledge' was indicated by score of $\leq 4$, while 'good knowledge' by a score of $\geq 5$. Similarly, 'poor attitude' was indicated by a score of $\leq 4$, while 'good attitude' by a score of 5 , and 'poor practice' was indicated by a score of $\leq 1$, while 'good practice' by a score of $\geq 2$.

The adopted BEVQ questionnaire investigates the frequency of plain water (bottle water/tap water) and SSB intake on a weekly basis. It consists of 10 types of beverages, including plain water, $100 \%$ fruit juice, sweetened fruit beverages, full-cream milk, low-fat milk, skimmed milk, regular soft drinks, energy and sports drinks, and alcoholic drinks. This questionnaire estimates the habitual intake of these beverages. The respondents were requested to indicate "how often" they consumed a beverage in the past month. There are seven categories of responses, ranging from "never or less than 1 time per week", to "more than two times per day". However, during analyses, we further simplified the categorisation into three primary categories, namely, "never or less than 1 time per week", " $\leq 6$ times per week" and "daily (1 time or more per day)". 
Anthropometric measurements such as body weight, height, waist circumference and body fat percentage were measured. Body weight was measured in light clothing, without shoes, using a portable digital electronic scale (Seca 813 digital electronic weighing scale). Height was measured as the distance from the top of the head to the bottom of the feet, without shoes, using a calibrated vertical stadiometer (Seca Portable 217). The BMI was calculated as kilogram body weight $/ \mathrm{m}^{2}$. The waist circumference was measured at the narrowest part, between the lower rib and the iliac crest, using a non-elastic flexible tape, and recorded to the nearest $0.1 \mathrm{~cm}$. The percentage of body fat was assessed using a bioelectrical impedance which consisted of a bipolar handheld device (Omron HBF-306, Omron, Japan), and the results were recorded as percentage of body weight. The subjects stood with their feet slightly separated, holding the device in both hands, arms stretched out at an angle of 90 degrees relative to the body, while the instrument recorded the impedance from hand to hand, and subsequently calculated the percentage of body fat to the nearest $0.1 \%$ based on age, gender, height and weight (16).

\section{Outcome measure}

The classification of BMI was based on the international obesity task force (IOTF) criteria. This criteria was used because it was shown to be most appropriate amongst the Asian adolescent population (17). Central obesity was defined by the waist circumference's $\geq 90$ th percentile, according to the waist circumference percentile curves for Malaysian children and adolescents. The cut-off value used was $83.8 \mathrm{~cm}$ for males, and $78.8 \mathrm{~cm}$ for females (18). The cut-off points for the normal body fat percentage was less than $30 \%$ for boys, and less than $35 \%$ for girls. This cut-off value is based on the body composition assessment for the development study of an international growth standard for preadolescent and adolescent children (19).

\section{Statistical Analysis}

The Statistical Package for Social Science (SPSS) version 21.0 was used for tabulation and analysis of the collected data. The demographic data was examined using descriptive statistics, and the results were reported as frequency and percentage values. The knowledge, attitude and practice (KAP) scores were calculated by summing up the participant's number of correct responses.

Scatter plots, skewness, and kurtosis were examined to determine the normality of the data distribution. Once the data distribution was determined, the not-normally distributed median was then taken. The assumption of logistic regression was then checked. The binary logistic regression analysis was carried out to verify the independent effects of the predictors on the dependent variables and predictors with a $p$ value $\leq 0.25$, which was entered in the multivariable logistic regression analysis model to identify the final predictors of KAPs, after controlling other independent variables. The odds ratio and $95 \% \mathrm{Cl}$ were calculated, and the result $(\mathrm{p} \leq 0.05)$ was considered statistically significant. The result was described in text form, before being summarized and presented in tables.

The frequency of plain water and SSB consumption were described via descriptive statistics, and the results were reported as a frequency and percentage. 


\section{Results}

\section{Socio-demographic characteristics and anthropometric profile of participants}

The socio-demographic characteristics of the participants are shown in Table 1. A total of 439 out of 450 respondents completed all of the questionnaires and measurements. The response rate was $97.6 \%$. The prevalence of being 'overweight' was $18.5 \%(n=81)$, while 'obesity' was $16.9 \%(n=74)$. The majority of the respondents $(73.8 \%, n=324)$ had a normal waist circumference level. However, slightly more than half of the respondents $(55.4 \%, n=243)$ had a normal body fat percentage. 
Table 1

Characteristics of respondents $(n=439)$

\begin{tabular}{|c|c|c|}
\hline Socio-demographic characteristics & Frequency (n) & Percentage (\%) \\
\hline Gender & 218 & 49.7 \\
\hline Male & 221 & 50.3 \\
\hline \multicolumn{3}{|l|}{ Female } \\
\hline Age & 381 & 86.8 \\
\hline $13-15$ years old & 58 & 13.2 \\
\hline \multicolumn{3}{|l|}{$16-17$ years old } \\
\hline Ethnicity & 422 & 96.1 \\
\hline Malay & 17 & 3.9 \\
\hline \multicolumn{3}{|l|}{ Others } \\
\hline Type of school & 242 & 55.1 \\
\hline Rural & 197 & 44.9 \\
\hline \multicolumn{3}{|l|}{ Urban } \\
\hline Father's occupation & 211 & 48.1 \\
\hline Professional & 228 & 51.9 \\
\hline \multicolumn{3}{|l|}{ Non-professional } \\
\hline Mother's occupation & 150 & 34.2 \\
\hline Professional & 289 & 65.8 \\
\hline \multicolumn{3}{|l|}{ Non-professional } \\
\hline Family monthly income & 52 & 11.8 \\
\hline$\leq \mathrm{RM} 1500$ & 117 & 26.7 \\
\hline RM 1501 - RM 2500 & 131 & 29.8 \\
\hline RM 2501 - RM 5000 & 72 & 16.4 \\
\hline RM 5001 - RM 8000 & 47 & 10.7 \\
\hline RM 8001 - RM 15,000 & 20 & 4.6 \\
\hline$\geq \operatorname{RM} 15,000$ & & \\
\hline
\end{tabular}




\begin{tabular}{|lll|}
\hline Socio-demographic characteristics & Frequency (n) & Percentage (\%) \\
\hline BMl-for-age classification & 39 & 8.88 \\
Underweight & 245 & 55.81 \\
Normal & 81 & 18.45 \\
Overweight & 74 & 16.86 \\
Obese & & \\
Waist circumference $(\mathrm{cm})$ & 324 & 73.80 \\
Normal & 115 & 26.20 \\
High & & \\
\hline Body fat percentage $(\%)$ & 243 & 54.36 \\
Normal & 196 & \\
\hline High & & 44.65 \\
\hline
\end{tabular}

\section{Mean score of knowledge, attitude and practice on SSB among participants}

The KAP score of the participants towards SSB intake was calculated separately, and presented in Table 2. Each correct response represents a score of 1 , and each wrong response represents a score of 0. Therefore, higher scores reflects better results. The highest mean percentage score was $88.43 \%$ for attitude, followed by a mean percentage score of $51.84 \%$ for knowledge, and the lowest mean score of $44.56 \%$ was for practice.

Table 2

The score of knowledge, attitude and practice on SSB

\begin{tabular}{|lll|}
\hline Variable & Mean (Percentage) & Median \\
\hline Knowledge Score & $5.18(51.84)$ & 5.00 \\
\hline Attitude Score & $4.42(88.43)$ & 5.00 \\
\hline Practice Score & $2.22(44.56)$ & 2.00 \\
\hline Maximum score: Knowledge: 10, Attitude: 5, Practice: 5. \\
\hline
\end{tabular}

Factors associated with participants' knowledge level on SSB

Amongst the variables entered in the bivariate analysis, only family monthly income has shown a significant association (Table 3). The family monthly income within the range of RM 1,501-RM 8,000 were more likely to get a good score for knowledge (score of $\geq 5$ ), with the best score in the range of 
family monthly income being RM 1,501- RM 2,500 (crude $\mathrm{OR}=0.50 ; 95 \% \mathrm{Cl}=0.26-0.98$ ). On the other hand, variables with a $p$-value $\leq 0.25$ which were entered in the multivariable logistic analysis, and some of the above associations, did not exist after adjustment for other variables. In the multivariable logistic analysis, subjects whose families earned an average monthly income of RM 1,501 - RM 2,500 were 0.51 times (adjusted OR $=0.51 ; 95 \% \mathrm{Cl}=0.26-0.99)$ and 0.45 times (RM $2501-\mathrm{RM} \mathrm{5000}$, adjusted OR = 0.45; $95 \% \mathrm{Cl}=0.22-0.92$ ) more likely to have an excellent knowledge on SSB, as compared to those who came from families who earned $\geq$ RM 5,000 a month. 
Table 3

Bivariable and multivariable logistic regression predicting knowledge on SSB among participants, $(\mathrm{N}=$ 439).

\begin{tabular}{|c|c|c|c|c|c|}
\hline & $\underset{5}{N}(\%)$ with score $\geq$ & $\begin{array}{l}\text { Crude OR }(95 \% \\
\mathrm{Cl})\end{array}$ & $\begin{array}{l}\mathrm{p}- \\
\text { value }\end{array}$ & $\begin{array}{l}\text { Adjusted } \\
\text { OR } \\
(95 \% \mathrm{Cl})\end{array}$ & $\begin{array}{l}\text { p- } \\
\text { value }\end{array}$ \\
\hline Gender & $148(67.9)$ & 1.00 & & 1.00 & \\
\hline Male & $146(66.1)$ & 1.09 & 0.684 & 1.16 & 0.483 \\
\hline Female & & $(0.73-1.62)$ & & $\begin{array}{l}(0.77- \\
1.74)\end{array}$ & \\
\hline Age & $250(65.6)$ & 1.00 & & 1.00 & \\
\hline $13-15$ & $44(75.9)$ & 0.61 & 0.125 & 0.66 & 0.224 \\
\hline $16-17$ & & $(0.32-1.15)$ & & $\begin{array}{l}(0.34- \\
1.29)\end{array}$ & \\
\hline \multirow{3}{*}{$\begin{array}{l}\text { Ethnicity } \\
\text { Malay } \\
\text { Others }\end{array}$} & $284(67.3)$ & 0.69 & 0.469 & 0.73 & 0.548 \\
\hline & & $(0.26-1.86)$ & & $\begin{array}{l}(0.27- \\
2.02)\end{array}$ & \\
\hline & $10(58.8)$ & 1.00 & & 1.00 & \\
\hline \multirow{3}{*}{$\begin{array}{l}\text { Type of school area } \\
\text { Urban }\end{array}$} & $134(68.0)$ & 1.00 & & 1.00 & \\
\hline & $160(66.1)$ & 1.09 & 0.673 & 1.14 & 0.553 \\
\hline & & $(0.73-1.63)$ & & $\begin{array}{l}(0.75- \\
1.73)\end{array}$ & \\
\hline Father's occupation & $144(68.2)$ & 1.00 & & 1.00 & \\
\hline Professional & $150(65.8)$ & 1.12 & 0.585 & 1.02 & 0.955 \\
\hline Non-professional & & $(0.75-1.67)$ & & $\begin{array}{l}(0.60- \\
1.71)\end{array}$ & \\
\hline \multirow{3}{*}{$\begin{array}{l}\text { Mother's occupation } \\
\text { Professional } \\
\text { Non-professional }\end{array}$} & 106 (70.7) & 1.00 & & 1.00 & \\
\hline & $188(65.1)$ & 1.29 & 0.236 & 1.21 & 0.457 \\
\hline & & $(0.85-1.98)$ & & $\begin{array}{l}(0.74- \\
1.97)\end{array}$ & \\
\hline \multirow{3}{*}{$\begin{array}{l}\text { Family monthly } \\
\text { income } \\
\leq \text { RM } 1500 \\
\text { RM } 1501 \text { - RM } 2500\end{array}$} & $27(51.9)$ & 1.00 & & 1.00 & \\
\hline & $80(68.4)$ & 0.50 & $0.042^{*}$ & 0.51 & $0.048 *$ \\
\hline & & $(0.26-0.98)$ & & $\begin{array}{l}(0.26- \\
0.99)\end{array}$ & \\
\hline
\end{tabular}




\begin{tabular}{|c|c|c|c|c|c|}
\hline $\begin{array}{l}\text { RM } 5001-\text { RM } 8000 \\
\text { RM } 8001 \text { - RM } \\
15,000\end{array}$ & $\underset{5}{N}(\%)$ with score $\geq$ & $\begin{array}{l}\text { Crude OR (95\% } \\
\mathrm{Cl})\end{array}$ & $\begin{array}{l}\mathrm{p}- \\
\text { value }\end{array}$ & $\begin{array}{l}\text { Adjusted } \\
\text { OR } \\
(95 \% \mathrm{Cl})\end{array}$ & $\begin{array}{l}\mathrm{p}- \\
\text { value }\end{array}$ \\
\hline \multirow[t]{4}{*}{$\geq \operatorname{RM} 15,000$} & $94(71.8)$ & $\begin{array}{l}0.43 \\
(0.22-0.83)\end{array}$ & $0.012^{\star}$ & $\begin{array}{l}0.45 \\
(0.22- \\
0.92)\end{array}$ & $0.029 *$ \\
\hline & $51(70.8)$ & $\begin{array}{l}0.45 \\
(0.21-0.94)\end{array}$ & $0.033^{\star}$ & $\begin{array}{l}0.51 \\
(0.21- \\
1.23)\end{array}$ & 0.135 \\
\hline & $32(68.1)$ & $\begin{array}{l}0.51 \\
(0.22-1.15)\end{array}$ & 0.104 & $\begin{array}{l}0.56 \\
\\
(0.21- \\
1.45)\end{array}$ & 0.230 \\
\hline & $10(50.0)$ & $\begin{array}{l}1.08 \\
(0.39-3.03)\end{array}$ & 0.884 & $\begin{array}{l}1.17 \\
(0.38- \\
3.64)\end{array}$ & 1.167 \\
\hline
\end{tabular}

\section{Factors associated with participant's attitude level on SSB}

In the bivariate analysis, two factors were significantly associated with a good attitude score (score $=5$ ) for SSB intake, which were the age category and type of school area, as shown in Table 4. In the attitude domain, participants aged between 16 to 17 years old showed a good attitude (crude OR $=0.5 ; 95 \% \mathrm{Cl}=$ $0.27-0.94)$, as compared to younger participants (13-15 years old). Participants at urban schools had the best score in the attitude level for SSB intake (crude OR $=1.71 ; 95 \% \mathrm{Cl}=1.16-2.54$ ), as compared to those in rural schools. In the multivariable logistic regression analysis, female participants had a score of 0.41 (adjusted $\mathrm{OR}=0.41,95 \% \mathrm{Cl}=0.27-0.62$ ) and participants aged between 16 to 17 years old had a score of 0.44 (adjusted $\mathrm{OR}=0.44,95 \% \mathrm{Cl}=0.22-0.85$ ), which were more likely to get a good attitude level for SSB. Participants from urban schools were 1.78 times (adjusted $\mathrm{OR}=1.78,95 \% \mathrm{Cl}=1.16-2.72$ ) were more likely to have a positive attitude on SSB intake compared to professional father's occupation. In addition, participants with parents who were not professionals (adjusted $O R=1.85 ; 95 \% \mathrm{Cl}=1.10-3.14$ ) were more likely to have a positive attitude toward SSB intake, compared to the parent's occupation. 
Table 4

Bivariable and multivariable logistic regression predicting attitude on SSB among participants, $(N=439)$.

\begin{tabular}{|c|c|c|c|c|c|}
\hline & $\mathrm{N}(\%)$ with score $=5$ & $\begin{array}{l}\text { Crude OR } \\
(95 \% \mathrm{Cl})\end{array}$ & p-value & $\begin{array}{l}\text { Adjusted OR } \\
(95 \% \mathrm{Cl})\end{array}$ & p-value \\
\hline Gender & $111(50.9)$ & 1.00 & & 1.00 & \\
\hline Male & $157(71.0)$ & 0.55 & 0.234 & 0.41 & $0.000 * * *$ \\
\hline Female & & $(0.21-1.46)$ & & $(0.27-0.62)$ & \\
\hline Age & $225(59.1)$ & 1.00 & & & \\
\hline $13-15$ & $43(74.1)$ & 0.50 & $0.030 *$ & 0.44 & $0.015^{\star}$ \\
\hline $16-17$ & & $(0.27-0.94)$ & & $(0.22-0.85)$ & \\
\hline Ethnicity & 260 (61.6) & 0.55 & 0.234 & 0.54 & 0.236 \\
\hline Malay & & $(0.21-1.46)$ & & $(0.19-1.51)$ & \\
\hline Others & $8(47.1)$ & 1.00 & & & \\
\hline Type of school area & $134(68.0)$ & 1.71 & $0.007^{\star \star}$ & 1.78 & $0.008^{* *}$ \\
\hline Urban & & $(1.16-2.54)$ & & $(1.16-2.72)$ & \\
\hline Rural & $134(55.4)$ & 1.00 & & 1.00 & \\
\hline Father's occupation & $133(63.0)$ & 1.00 & & 1.00 & \\
\hline Professional & 135 (59.2) & 1.18 & 0.412 & 1.85 & $0.022^{\star}$ \\
\hline Non-professional & & $(0.80-1.73)$ & & $(1.10-3.14)$ & \\
\hline Mother's occupation & $91(60.7)$ & 1.00 & & 1.00 & \\
\hline Professional & 135 (59.2) & 0.98 & 0.906 & 1.08 & 0.759 \\
\hline Non-professional & & $(0.65-1.46)$ & & $(0.66-1.75)$ & \\
\hline Family monthly income & $31(59.6)$ & 1.00 & & 1.00 & \\
\hline$\leq \mathrm{RM} 1500$ & $74(63.2)$ & 0.86 & 0.653 & 0.96 & 0.904 \\
\hline RM 1501 - RM 2500 & & $(0.44-1.68)$ & & $(0.47-1.93)$ & \\
\hline RM 2501 - RM 5000 & $87(66.4)$ & 0.75 & 0.387 & 0.98 & 0.952 \\
\hline RM 5001 - RM 8000 & & $(0.39-1.45)$ & & $(0.46-2.06)$ & \\
\hline \multicolumn{6}{|c|}{ 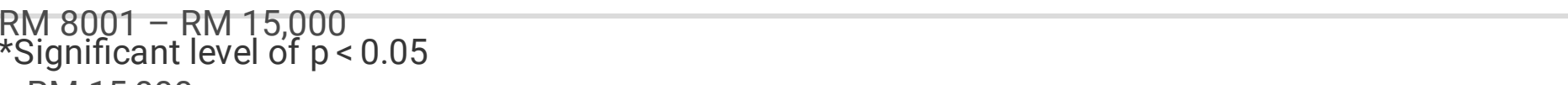 } \\
\hline$\star \star \star$ Significant level of $p$ & 001 & & & & \\
\hline
\end{tabular}




\begin{tabular}{|c|c|c|c|c|c|}
\hline & $\mathrm{N}(\%)$ with score $=5$ & $\begin{array}{l}\text { Crude OR } \\
(95 \% \mathrm{Cl})\end{array}$ & p-value & $\begin{array}{l}\text { Adjusted OR } \\
(95 \% \mathrm{Cl})\end{array}$ & p-value \\
\hline & \multirow[t]{2}{*}{$38(52.8)$} & 1.32 & \multirow[t]{2}{*}{0.450} & 2.28 & \multirow[t]{2}{*}{0.072} \\
\hline & & $(0.64-2.72)$ & & $(0.93-5.57)$ & \\
\hline & \multirow[t]{2}{*}{$29(61.7)$} & 0.92 & \multirow[t]{2}{*}{0.832} & 1.42 & \multirow[t]{2}{*}{0.480} \\
\hline & & $(0.41-2.06)$ & & $(0.54-3.78)$ & \\
\hline & \multirow[t]{2}{*}{$9(45.0)$} & 1.80 & \multirow[t]{2}{*}{0.266} & 1.96 & \multirow[t]{2}{*}{0.258} \\
\hline & & $(0.64-5.11)$ & & $(0.61-6.32)$ & \\
\hline \multicolumn{6}{|c|}{ *Significant level of $p<0.05$} \\
\hline \multicolumn{6}{|c|}{$\star \star$ Significant level of $p<0.01$} \\
\hline$\star * \star$ Significant level of $p<$ & .001 & & & & \\
\hline
\end{tabular}

\section{Factors associated with participant's practice level on SSB}

With regards to the practice score, there were no associations between the socio-demographic characteristics in the bivariate logistic regression analysis, as shown in Table 5. 
Table 5

Bivariable and multivariable logistic regression predicting practice on SSB among participants, $(N=439)$.

\begin{tabular}{|c|c|c|c|c|c|}
\hline & $\underset{2}{N}(\%)$ with score $\geq$ & $\begin{array}{l}\text { Crude OR } \\
(95 \% \mathrm{Cl})\end{array}$ & $\begin{array}{l}\text { p- } \\
\text { value }\end{array}$ & $\begin{array}{l}\text { Adjusted OR } \\
(95 \% \mathrm{Cl})\end{array}$ & p-value \\
\hline \multirow{3}{*}{$\begin{array}{l}\text { Gender } \\
\text { Male } \\
\text { Female }\end{array}$} & $153(70.2)$ & 1.00 & & 1.00 & \\
\hline & $168(76.0)$ & 0.74 & 0.169 & 0.73 & 0.160 \\
\hline & & $(0.49-1.13)$ & & $(0.48-1.13)$ & \\
\hline \multirow{3}{*}{$\begin{array}{l}\text { Age } \\
13-15 \\
16-17\end{array}$} & $284(74.5)$ & 1.00 & & 1.00 & \\
\hline & $37(63.8)$ & 1.66 & 0.088 & 1.78 & 0.065 \\
\hline & & $(0.93-2.98)$ & & $(0.96-3.28)$ & \\
\hline \multirow{3}{*}{$\begin{array}{l}\text { Ethnicity } \\
\text { Malay } \\
\text { Others }\end{array}$} & 305 (72.3) & 6.14 & 0.080 & 6.12 & 0.082 \\
\hline & & $\begin{array}{l}(0.81- \\
46.80)\end{array}$ & & $(0.80-47.12)$ & \\
\hline & $16(94.1)$ & 1.00 & & 1.00 & \\
\hline \multirow{3}{*}{$\begin{array}{l}\text { Type of school area } \\
\text { Urban }\end{array}$} & $143(72.6)$ & 1.00 & & 1.00 & \\
\hline & $178(73.6)$ & 0.95 & 0.821 & 0.96 & 0.839 \\
\hline & & $(0.62-1.46)$ & & $(0.61-1.49)$ & \\
\hline \multirow{3}{*}{$\begin{array}{l}\text { Father's occupation } \\
\text { Professional } \\
\text { Non-professional }\end{array}$} & $152(72.0)$ & 1.00 & & 1.00 & \\
\hline & $169(74.1)$ & 0.90 & 0.623 & 0.73 & 0.244 \\
\hline & & $(0.59-1.37)$ & & $(0.42-1.25)$ & \\
\hline \multirow{3}{*}{$\begin{array}{l}\text { Mother's occupation } \\
\text { Professional } \\
\text { Non-professional }\end{array}$} & 107 (71.3) & 1.00 & & 1.00 & \\
\hline & $214(74.0)$ & 0.87 & 0.543 & 0.86 & 0.569 \\
\hline & & $(0.56-1.36)$ & & $(0.51-1.44)$ & \\
\hline \multirow{2}{*}{$\begin{array}{l}\text { Family monthly } \\
\text { income }\end{array}$} & 37 (71.2) & 1.00 & & 1.00 & \\
\hline & $85(72.6)$ & 0.93 & 0.841 & 0.86 & 0.680 \\
\hline RM 1501 - RM 2500 & & $(0.45-1.92)$ & & $\begin{array}{l}(0.408- \\
1.797)\end{array}$ & \\
\hline RM 2501 - RM 5000 & $96(73.3)$ & 0.90 & 0.771 & 0.71 & 0.404 \\
\hline RM 5001 - RM 8000 & & $(0.44-1.84)$ & & $(0.32-1.57)$ & \\
\hline
\end{tabular}

RM 8001 - RM 15,000

$\geq \operatorname{RM} 15,000$ 


\begin{tabular}{|llllll|}
\hline $\mathbf{2}(\%)$ with score $\geq$ & $\begin{array}{l}\text { Crude OR } \\
(\mathbf{9 5 \%} \mathrm{Cl})\end{array}$ & $\begin{array}{l}\mathbf{p} \text { - } \\
\text { value }\end{array}$ & $\begin{array}{l}\text { Adjusted OR } \\
(\mathbf{9 5 \%} \mathrm{Cl})\end{array}$ & p-value \\
\hline $53(73.6)$ & 0.88 & 0.762 & 0.59 & 0.273 \\
& $(0.40-1.96)$ & & $\begin{array}{l}(0.228- \\
1.519)\end{array}$ & \\
\hline $36(76.6)$ & 0.75 & 0.540 & 0.50 & 0.195 \\
& $(0.31-1.86)$ & & $(0.18-1.43)$ & \\
\hline $14(70.0)$ & 1.06 & 0.923 & 0.73 & 0.727 \\
& $(0.34-3.27)$ & & $(0.21-2.52)$ & \\
\hline
\end{tabular}

\section{The frequency of beverage intake of participants}

Table 6 shows the frequency of beverage intake by the participants. On a daily basis, plain water (84.3\%) was the most common beverage intake by the respondents, followed by sweetened coffee or tea (25.5\%) and full cream milk (16.6\%). Thus, we can conclude that, among the respondents, at least one-quarter of them consumed SSB on a daily basis. More than half $(60 \%)$ of the participants consumed SSB less than once a week. Alcoholic drinks had the lowest frequency intake, with $98.9 \%$ of the participants consuming such beverages either once a week, or not at all. This low intake may be due to religious reasons, since the majority of the participants were Muslims. 
Table 6

Frequency of beverages consumption $(n=439)$

\begin{tabular}{|llll|}
\hline Types of beverages & Never or $\leq 1$ time per week & $\begin{array}{l}\leq 6 \text { times per week } \\
\mathbf{n}(\%)\end{array}$ & $\begin{array}{l}\text { Daily } \\
\mathbf{n}(\%)\end{array}$ \\
\hline Plain water & $14(3.2)$ & $55(12.5)$ & $370(84.3)$ \\
\hline 100\% Fruit Juice & $287(65.4)$ & $118(26.9)$ & $34(7.7)$ \\
\hline Sweetened Fruit Beverage & $246(56.1)$ & $149(33.9)$ & $44(10.0)$ \\
\hline Full cream milk & $205(46.7)$ & $161(36.7)$ & $73(16.6)$ \\
\hline Low Fat Milk (2\%) & $271(61.7)$ & $113(25.8)$ & $55(12.5)$ \\
\hline Skimmed milk & $342(77.9)$ & $59(13.5)$ & $38(8.7)$ \\
\hline Regular Soft Drinks & $287(65.4)$ & $118(26.9)$ & $34(7.7)$ \\
\hline Sweetened coffee or tea & $117(26.7)$ & $210(47.8)$ & $112(25.5)$ \\
\hline Energy \& Sports Drinks & $277(63.1)$ & $115(26.2)$ & $47(10.7)$ \\
\hline Alcoholic drinks & $434(98.9)$ & $2(0.5)$ & $3(0.7)$ \\
\hline Other beverages & $419(95.4)$ & $15(3.4)$ & $5(1.2)$ \\
\hline
\end{tabular}

\section{The association between KAP on SSB with body composition}

Table 7 shows the association between KAPs on SSB intake, with regards to body composition. No association was found between KAPs on SSB intake, with that of body mass index, and waist circumference level. However, there was a significant association $(p<0.05)$ found between KAP on SSB intake, and body fat percentage. The participants who had a high body fat percentage resulted in a much higher attitude percentage score. 
Table 7

Mean \pm SD of KAP and body composition

\begin{tabular}{|llll|}
\hline Types of beverages & Knowledge & Attitude & Practice \\
\hline BMI & $p=0.449$ & $p=0.198$ & $p=0.407$ \\
Underweight $(n=39)$ & $50.26 \pm 16.14$ & $84.62 \pm 24.48$ & $40.51 \pm 24.06$ \\
Normal $(n=245)$ & $51.51 \pm 16.59$ & $87.76 \pm 17.93$ & $44.49 \pm 23.42$ \\
Overweight $(n=81)$ & $51.23 \pm 14.70$ & $91.60 \pm 14.79$ & $47.65 \pm 20.34$ \\
Obese $(n=74)$ & $51.46 \pm 13.96$ & $89.19 \pm 18.19$ & $43.51 \pm 21.80$ \\
Body Fat Percentage & $p=0.992$ & $p=0.013^{*}$ & $p=0.381$ \\
Normal $(n=243)$ & $51.85 \pm 16.82$ & $86.50 \pm 19.64$ & $43.70 \pm 23.45$ \\
High $(n=196)$ & $51.84 \pm 14.45$ & $90.81 \pm 15.86$ & $45.61 \pm 21.70$ \\
\hline Waist circumference & $p=0.419$ & $p=0.435$ & $p=0.243$ \\
Normal $(n=324)$ & $51.48 \pm 16.04$ & $88.02 \pm 18.88$ & $45.30 \pm 23.00$ \\
High $(n=115)$ & $52.87 \pm 15.09$ & $89.57 \pm 15.97$ & $42.43 \pm 21.71$ \\
\hline *Significant difference between the mean $(p<0.05)$ by ANOVA test. \\
\hline
\end{tabular}

\section{Discussion}

The findings revealed a higher level of attitude, moderate level of knowledge, and poor level of lifestyle choices amongst SSB intake for the participants. The positive attitudes show that the participants were more attentive toward reducing the SSB consumption. In general, they were aware of the needs to read the food labels and ingredients, and to choose beverages without simple sugars. This shows that, although they had a good attitude towards SSB intake, it did not change the way they limited this intake. This is in agreement prior work (20), which suggests that these positive attitudes are neither preventive, nor health-promoting. As the level of knowledge was considered relatively low, this may reflect the low level of awareness amongst the participants in practicing good habits associated with avoiding SSBs and limiting their intake in general.

There was an association between knowledge levels on SSBs, and monthly family income of the participants. It was reported that low level family monthly income groups were a contributing factor toward having excellent knowledge on SSB, as compared to those who earned $\geq$ RM 5,000 as a monthly family income. Income-related factors have previously been described as factors that attribute to inequalities of knowledge in nutrition (21). This study brought forward interesting findings that demonstrate that those with low income also reflected good knowledge in SSBs. This finding is in agreement with the suggestion by Xu et al. (2020), who mention that the inequality of knowledge is also strongly related to geographical areas with good accessibility to education facilities (22). Hence, the area 
considered in this study has good accessibility to facilities such as well-equipped public educational institutions and also multiple areas with free internet hot spots. All these knowledge related sources can be gained by anyone, without any additional cost. However, another important issue that needs to be addressed is that this knowledge cannot be translated into practice by those families with limited income, as they do not have the option to buy healthier foods such as fresh fruits and vegetables. The concern was more on satisfying hunger, and to fulfil the desire of food with good taste. Therefore, there was a higher tendency to buy high sugar foods and SSBs (23). It was also shown that parental influence on the intake of a child's drinks may be linked to the knowledge associated with diet and health (24). Based on previous studies for children and adolescents, it was shown that the availability of SSBs was correlated with the child's intake, which was much better correlated amongst well-educated and high income parents (25). The participants who had a high income could explain that they afforded to buy SSBs, which led to their children consuming more SSBs, as compared to low income level groups. As in agreement with a recent systematic review (26), we also found that neither level of education nor income have any association with good practices in SSBs intake.

This study discovered that gender, age and location of school impact the overall attitude towards SSB intake. Female with an older age, living in urban areas were found to have a high awareness (attitude) towards SSB intake. Similar observations have also been reported in previous studies amongst Australians (27). Our findings also support previous studies showing that boys had a higher intake of SSBs than girls (28). Females may respond more favourably to these efforts, as they appear to be more concerned about body weight issues, and are more likely to rate behaviours of food choice as an essential means for their life, as compared to males (29). The same goes to the participants who were from urban schools, as they had a good attitude, compared to those who were from the rural schools. This is because rural residents have many characteristics, including widespread socioeconomic disadvantages, and can easily access unhealthy foods that increase their risk for chronic diseases, food insecurity, poor dietary behaviours, and higher intake of SSBs (30).

The results by this study also demonstrate that $19 \%$ of the participants were overweight, and $17 \%$ were obese. This shows that one-third of the participants fall between the overweight and obese categories, which reflects the high rate of overweight and obesity issues amongst the adolescents. These findings were almost similar to those from a related study that was also conducted amongst adolescents in Malaysia (31). The findings of this study also found that the average waist circumference measurements of the respondents was $74 \mathrm{~cm}$ for boys, and $75 \mathrm{~cm}$ for girls. In a study conducted by Higgins et al. (32), it was stated that children with a waist circumference level of $71 \mathrm{~cm}$ or more, were 14 times more likely to develop a negative risk profile than a normal one. Therefore, $26 \%$ of the boys and girls in this study had a high waist circumference measurement, and were more likely to develop a negative risk profile. The findings also show that nearly half of the participants had a high body fat percentage, which is associated with a high risk of diseases such as hypertension, type 2 diabetes, cardiovascular diseases and metabolic syndromes (33). 
The findings of this study pointed out that the most frequently consumed beverage on a daily basis was plain water. This finding is similar to that in the study by Zahran (34), in which most of their participants consumed plain water on a daily basis. Tea and caffeinated drinks $(25.5 \%)$ were the most consumed SSBs on a daily basis amongst the participants in the present study. This trend is similar to studies by Norimah et al. (35) and Teng et al. (36), which reported a high consumption of coffee and tea amongst their participants. These findings are in agreement with a study that demonstrated that the reasons behind the high consumption of coffee and tea amongst students included helping them to concentrate, keep them awake at night during exam preparation phases, and stay active during classes (37). The majority of the participants either never drank SSB, or consumed it less than once a week. This reflects that this particular population sample consumed SBBs occasionally rather than regularly. Alcoholic drinks were the least consumed beverages. This is because of religious issues, since most of the participants were Muslims, and all Muslims are prohibited from consuming alcoholic drinks. Further statistical analyses could not find any significant association between the SSB consumption patterns, and KAP levels. However, we found that those participants with a high body fat percentage did have a high attitude percentage score.

There were a few limitations in this study. Firstly, the data for beverages intake in the survey was selfreported. Self-reported data has the potential of being underreported in terms of beverage consumption, especially for overweight or obese children, due to social desirability bias and other factors. While exploring the associations between SSB and body composition, other dietary, physical, genetic and environmental factors that could also impact the value of the body composition, were not studied.

Nevertheless, our findings have implications on future research. As we found a reasonable gap between knowledge, attitude and practices (KAPs), it is necessary to develop or strengthen strategies by which positive attitudes can be converted into promising practices. The education towards improvement of knowledge of SSBs should be enhanced in the education syllabus. It is recommended to implement this strategy by taking into consideration the socioeconomic status, as such emphasizing on the nutrition information about SSBs as well as healthy eating among participants belonging to high income families. Attempts should also be made to reinforce the consequences of high SSB intake in order to transform them to positive practices. The availability of SSBs should be minimised, both in school and at home, to support healthier lifestyle changes.

\section{Conclusion}

This study examined the associated factors that determine the knowledge, attitude and practices (KAPs) of adolescents towards SSBs. The results indicate that, amongst adolescents in secondary schools across Selangor, there was a high level of attitude and moderate level of knowledge, but low level of lifestyle choices, toward sugar-sweetened beverages (SSBs). A high attitude was mostly observed amongst those with a high body fat percentage. 
The interventions associated with increasing knowledge and awareness towards the consequences of SSB intake should be carried out. The strategy of those interventions must include strategies to transform the knowledge received into healthier lifestyle changes by limiting the consumption of SBBs by adolescents.

\section{Declarations}

\section{Ethics approval and consent to participate}

This study was conducted according to the guidelines laid down in the Declaration of Helsinki and all procedures involving research study participants were approved by the Research Ethics Committee of Universiti Teknologi MARA, Malaysia. Written informed consent was obtained from all subjects.

\section{Consent for publication}

Not applicable

\section{Availability of data and materials}

Data sharing not applicable to this article as no datasets were generated or analysed during the current study

\section{Competing interests}

The authors declare that they have no competing interests

\section{Funding}

The authors declare that no funding received for this study

\section{Author's contributions}

All of the authors participated in the study design. Nur Liyana Izlin \& Nur Zulaikha Semaon collected the data, performed the data analyses, and drafted the manuscript. Nur Islami Mohd Fahmi Teng and Norsham Juliana Nordin were responsible for designed the study, data analyses and critically revising the manuscript. All authors contributed to the writing and approval of the final version of the manuscript.

\section{Acknowledgement}

We would like to thank all the teachers and students who helped us throughout the data collection phase. We are also grateful for the support and guidance provided by our colleagues and lecturers from the Faculty of Health Sciences, UiTM Puncak Alam.

\section{Author's information}


NIMFT, the corresponding author is currently the lead of MiCHILD (Maternal, Infant and Young Child) Nutrition research group in Universiti Teknologi MARA.

\section{References}

1. Van der Horst K, Kremers S, Ferreira I, Singh A, Oenema A, Brug J. Perceived parenting style and practices and the consumption of sugar-sweetened beverages by adolescents. Health Educ Res. 2007;22(2):295-304.

2. Abdullah NN, Mokhtar MM, Bakar MHA, Al-Kubaisy W. Trend on fast food consumption in relation to obesity among selangor urban community. Procedia-Social Behavioral Sciences. 2015;202:505-13.

3. Ahmad A, Zulaily N, Shahril MR, Abdullah EFHS, Ahmed A. Association between socioeconomic status and obesity among 12-year-old Malaysian adolescents. PloS one. 2018;13(7).

4. Chee Cheong K, Yoon Ling C, Kuang Hock L, Mohd Ghazali S, Chien Huey T, Ibrahim C, et al. Association between Availability of Neighborhood Fast Food Outlets and Overweight Among 5-18 Year-Old Children in Peninsular Malaysia: A Cross-Sectional Study. Int J Environ Res Public Health. 2019;16(4):593.

5. Wee BS, Poh BK, Bulgiba A, Ismail MN, Ruzita AT, Hills AP. Risk of metabolic syndrome among children living in metropolitan Kuala Lumpur: a case control study. BMC Public Health. 2011;11(1):333.

6. Lin G, Scott J. NIH Public Access. 2012;100(2):130-4.

7. Kosova EC, Auinger P, Bremer AA. The relationships between sugar-sweetened beverage intake and cardiometabolic markers in young children. Journal of the Academy of Nutrition Dietetics. 2013;113(2):219-27.

8. Malik VS, Popkin BM, Bray GA, Després J-P, Willett WC, Hu FB. Sugar-sweetened beverages and risk of metabolic syndrome and type 2 diabetes: a meta-analysis. Diabetes Care. 2010;33(11):2477-83.

9. Amarra MSV, Khor GL, Chan P. Intake of added sugar in Malaysia: a review. Asia Pac J Clin Nutr. 2016;25(2):227-40.

10. Abdul Majid H, Ramli L, Ying SP, Su TT, Jalaludin MY, Mohsein A, et al. Dietary Intake among Adolescents in a Middle-Income Country: An Outcome from the Malaysian Health and Adolescents Longitudinal Research Team Study (the MyHeARTs Study). PloS one. 2016;11(5).

11. Pengpid S, Peltzer K. High Carbonated Soft Drink Intake is Associated with Health Risk Behavior and Poor Mental Health among School-Going Adolescents in Six Southeast Asian Countries. International Journal of Environmental Research Public Health. 2020;17(1):132.

12. Department of statistics Malaysia official portal. The source of Malaysia's official statistics Putrajaya, Malaysia2020 [cited 20205 May 2020]. Selangor]. Available from: https://www.dosm.gov.my/v1/index.php? $r=$ column/cone\&menu_id=eGUyTm9RcEVZSIImYW45dmpnZHh4dz0. 
13. Teng NIMF, Nordin NJ, Latif SZA. Development and validation of a questionnaire assessing the knowledge, attitudes and practices of young adults in Malaysia towards sugar sweetened beverages. International Journal of Research in Pharmaceutical Sciences. 2019;10(4):2992-9.

14. Hedrick VE, Savla J, Comber DL, Flack KD, Estabrooks PA, Nsiah-Kumi PA, et al. Development of a brief questionnaire to assess habitual beverage intake (BEVQ-15): sugar-sweetened beverages and total beverage energy intake. Journal of the Academy of Nutrition Dietetics. 2012;112(6):840-9.

15. Teng NIMF, Nordin NJ, Abd Latif SZ. Development and validation of a questionnaire assessing the knowledge, attitudes and practices of young adults in Malaysia towards sugar sweetened beverages. International Journal of Research in Pharmaceutical Sciences. 2019;10(4):2992-9.

16. Gartner A, Dioum A, Delpeuch F, Maire B, Schutz Y. Use of hand-to-hand impedancemetry to predict body composition of African women as measured by air displacement plethysmography. Eur $\mathrm{J}$ Clin Nutr. 2004;58(3):523-31.

17. Viner R, Fry T, Gupta S, Kinra S, McCarthy D, Saxena S, et al. Insufficient evidence to support separate BMI definitions for obesity in children and adolescents from south Asian ethnic groups in the UK. International Journal of obesity. 2010;34(4):656-8.

18. Poh BK, Jannah AN, Chong LK, Ruzita AT, Ismail MN, McCarthy D. Waist circumference percentile curves for Malaysian children and adolescents aged 6.0-16.9 years. International Journal of Pediatric Obesity. 2011;6(3-4):229-35.

19. Lohman TG, Going SB. Body composition assessment for development of an international growth standard for preadolescent and adolescent children. Food nutrition bulletin. 2006;27(4_suppl5):314S25.

20. Shafi T, Aman Z. Knowledge Attitude \& Practices (KAP) Regarding Carbonated Drinks among female medical students of Allama Iqbal Medical College, Lahore. Proceeding SZPGMI Vol. 2017;31(2):92 7.

21. Cluss PA, Ewing L, King WC, Reis EC, Dodd JL, Penner B. Nutrition knowledge of low-income parents of obese children. Translational behavioral medicine. 2013;3(2):218-25.

22. Xu Y, Zhu S, Zhang T, Wang D, Hu J, Gao J, et al. Explaining Income-Related Inequalities in Dietary Knowledge: Evidence from the China Health and Nutrition Survey. International Journal of Environmental Research Public Health. 2020;17(2):532.

23. Fielding-Singh P. A Taste of Inequality: Food's Symbolic Value across the Socioeconomic Spectrum. Sociological Science. 2017;4.

24. Zahid A, Davey C, Reicks M. Beverage intake among children: associations with parent and homerelated factors. Int J Environ Res Public Health. 2017;14(8):929.

25. Bjelland M, Lien N, Grydeland M, Bergh IH, Anderssen SA, Ommundsen Y, et al. Intakes and perceived home availability of sugar-sweetened beverages, fruit and vegetables as reported by mothers, fathers and adolescents in the HEIA (HEalth In Adolescents) study. Public Health Nutr. 2011;14(12):2156-65.

26. Hinnig PdF, Monteiro JS, De Assis MAA, Levy RB, Peres MA, Perazi FM, et al. Dietary patterns of children and adolescents from high, medium and low human development countries and associated 
socioeconomic factors: a systematic review. Nutrients. 2018;10(4):436.

27. Miller C, Wakefield M, Braunack-Mayer A, Roder D, O'Dea K, Ettridge K, et al. Who drinks sugar sweetened beverages and juice? An Australian population study of behaviour, awareness and attitudes. BMC obesity. 2019;6(1):1.

28. Park S, Blanck HM, Sherry B, Brener N, O'Toole T. Factors associated with sugar-sweetened beverage intake among United States high school students. J Nutr. 2012;142(2):306-12.

29. Ranjit N, Evans MH, Byrd-Williams C, Evans AE, Hoelscher DM. Dietary and activity correlates of sugar-sweetened beverage consumption among adolescents. Pediatrics. 2010;126(4):e754-e61.

30. Sharkey JR. Measuring potential access to food stores and food-service places in rural areas in the US. Am J Prev Med. 2009;36(4):151-S5.

31. Gan WY, Mohamed SF, Law LS. Unhealthy Lifestyle Associated with Higher Intake of SugarSweetened Beverages among Malaysian School-Aged Adolescents. Int J Environ Res Public Health. 2019;16(15):2785.

32. Higgins PB, Gower BA, Hunter GR, Goran MI. Defining health-related obesity in prepubertal children. Obes Res. 2001;9(4):233-40.

33. Reilly JJ, Methven E, McDowell ZC, Hacking B, Alexander D, Stewart L, et al. Health consequences of obesity. Arch Dis Child. 2003;88(9):748-52.

34. Zahran SM. Association of beverage consumption with obesity in healthy adults. Arab Journal of Nutrition and Exercise (AJNE). 2016:37-51.

35. Norimah JA, Safiah M, Jamal K, Haslinda S, Zuhaida H, Rohida S, et al. Food Consumption Patterns: Findings from the Malaysian Adult Nutrition Survey (MANS). Malaysian Journal of Nutrition. 2008;14(1):25-39.

36. Teng NIMF, Nordin NJ, Suraya A, Shah M. Plain water and beverage consumption patterns among university students in Puncak Alam, Malaysia. Peer-reviewed Journal of the Nutrition Society of Malaysia. 2019;25(2):227.

37. Santacroce R, Martinotti G, Cinosi E, Lupi M, Acciavatti T, Marini S, et al. Alcohol, coffee and energy drink consumption patterns in a sample of Italian adolescents and young adults! Health. 2014. 